The University of Southern Mississippi

The Aquila Digital Community

Faculty Publications

7-2016

\title{
Electrons and Phonons in Amorphous Semiconductors
}

\author{
Kiran Prasai \\ Ohio University \\ Parthapratim Biswas \\ University of Southern Mississippi \\ D.A. Drabold \\ Ohio University
}

Follow this and additional works at: https://aquila.usm.edu/fac_pubs

\section{Recommended Citation}

Prasai, K., Biswas, P., Drabold, D. (2016). Electrons and Phonons in Amorphous Semiconductors. Semiconductor Science and Technology, 31(7).

Available at: https://aquila.usm.edu/fac_pubs/17455

This Article is brought to you for free and open access by The Aquila Digital Community. It has been accepted for inclusion in Faculty Publications by an authorized administrator of The Aquila Digital Community. For more information, please contact Joshua.Cromwell@usm.edu. 


\section{TOPICAL REVIEW}

\section{Electrons and phonons in amorphous semiconductors}

To cite this article: Kiran Prasai et al 2016 Semicond. Sci. Technol. 31073002

\section{Manuscript version: Accepted Manuscript}

Accepted Manuscript is "the version of the article accepted for publication including all changes made as a result of the peer review process, and which may also include the addition to the article by IOP Publishing of a header, an article ID, a cover sheet and/or an 'Accepted Manuscript' watermark, but excluding any other editing, typesetting or other changes made by IOP Publishing and/or its licensors"

This Accepted Manuscript is (c) (c) 2016 IOP Publishing Ltd.

During the embargo period (the 12 month period from the publication of the Version of Record of this article), the Accepted Manuscript is fully protected by copyright and cannot be reused or reposted elsewhere.

As the Version of Record of this article is going to be / has been published on a subscription basis, this Accepted Manuscript is available for reuse under a CC BY-NC-ND 3.0 licence after the 12 month embargo period.

After the embargo period, everyone is permitted to use copy and redistribute this article for non-commercial purposes only, provided that they adhere to all the terms of the licence https://creativecommons.org/licences/by-nc-nd/3.0

Although reasonable endeavours have been taken to obtain all necessary permissions from third parties to include their copyrighted content within this article, their full citation and copyright line may not be present in this Accepted Manuscript version. Before using any content from this article, please refer to the Version of Record on IOPscience once published for full citation and copyright details, as permissions will likely be required. All third party content is fully copyright protected, unless specifically stated otherwise in the figure caption in the Version of Record.

View the article online for updates and enhancements. 
Electrons and Phonons in Amorphous Semiconductors

\author{
Kiran Prasai, ${ }^{1}$ Parthapratim Biswas, ${ }^{2}$ and D. A. Drabold ${ }^{1}$ \\ ${ }^{1}$ Department of Physics and Astronomy, Ohio University, Athens OH 45701, USA \\ ${ }^{2}$ Department of Physics and Astronomy, The University of Southern Mississippi, Hattiesburg MS 39406, USA
}

(Dated: April 27, 2016)

\begin{abstract}
The coupling between lattice vibrations and electrons is one of the central concepts of condensed matter physics. The subject has been deeply studied for crystalline materials, but far less so for amorphous and glassy materials, which are among the most important for applications. In this paper, we explore the electron-lattice coupling using current tools of first-principles computer simulation. We choose three materials to illustrate the phenomena: amorphous silicon ( $a-\mathrm{Si}$ ), amorphous selenium $(a-\mathrm{Se})$ and amorphous gallium nitride $(a-\mathrm{GaN})$. In each case, we show that there is a strong correlation between the localization of electron states and the magnitude of thermallyinduced fluctuations in energy eigenvalues obtained from the density-functional theory (i.e. KohnSham eigenvalues). We provide a heuristic theory to explain these observations. The case of $a$-GaN, a topologically disordered partly ionic insulator, is distinctive compared to the covalent amorphous examples. Next, we explore the consequences of changing the charge state of a system as a proxy for tracking photo-induced structural changes in the materials. Where transport is concerned, we lend insight into the Meyer-Neldel compensation rule and discuss a thermally averaged Kubo-Greenwood formula as a means to estimate electrical conductivity and especially its temperature dependence. We close by showing how the optical gap of an amorphous semiconductor can be computationally engineered with the judicious use of Hellmann-Feynman forces (associated with a few defect states) using molecular dynamics simulations. These forces can be used to close or open an optical gap, and identify a structure with a prescribed gap. We use the approach with plane-wave density functional methods to identify a low-energy amorphous phase of silicon including several coordination defects, yet with a gap near that of good quality a-Si models.
\end{abstract}

\section{INTRODUCTION}

Amorphous semiconductors and glasses are among the most important materials, with diverse technological applications. Man-made materials have been at our side since at least the middle of the fourth millennium BC, initially in the form of glazed quartz or faience. Despite this long history of glass-making, our understanding of physico-chemical properties of glasses or amorphous solids in general from a microscopic point of view only began in the 1960s. Electronic applications of amorphous semiconductors have burgeoned, with utilization for electricity generation [1], night-vision applications [2], thinfilm transistors [3], computer-memory technologies [4], fiber-optic "light pipes" [5] and many others beside. In this paper, we focus on one substantial issue - the interplay between the electronic properties (electronic states and energies) and the atomic coordinates (and associated motion of the atoms when in or out of thermal equilibrium). This relationship between the electronic and ionic degrees of freedom is described by the electron-phonon coupling (EPC), which plays a crucial role in determining thermal and optical responses of a solid to light and charge-carrier transport.

Amorphous materials lack long-range structural order. This makes them difficult to study via conventional approaches, and enables physical attributes unknown in crystals. The vibrations are distinctive compared to the crystalline case, with motion less "confined" than that for a crystal. This "dynamical sloppiness" [6] manifests itself in the curious observation that there are thermally induced defect fluctuations - thermal dynamics are sufficient to change the instantaneous population of under or over coordinated atoms. On much lower energy scales, we have also observed that the nature of the amorphous vibrational ground state is ambiguous [7]. This dynamical sloppiness inevitably influences the electronic structure and is naturally addressed through the EPC.

For both electrons and phonons, the wave functions and classical normal modes may be localized, i.e. confined only to a finite volume of space. This is in contrast to any crystals, for which Bloch's Theorem applies, and it is clear that all electron or vibrational states are extended (though of course not necessarily uniformly so). The localized states occur in spectral gaps in the electronic or vibrational density of states. Any physical process that involves these localized states will be markedly different than for the extended states of translationally invariant systems. The electronic structure of disordered materials may be addressed by adopting a chemist's viewpoint to bonding between atoms or molecules in real space. The local electronic density of states (DOS) is computed from the diagonal elements of the density matrix, whereas the off-diagonal elements provide useful information about the locality of interatomic interactions ${ }^{1}$.

The density of states of amorphous solids bears some resemblance to their crystalline counterparts. For example, there is a spectral gap for electrons in both crys-

\footnotetext{
${ }^{1}$ For realistic calculations of the decay of the density matrix and generalized Wannier functions, see Ref. [8-10]
} 
talline and amorphous semiconductors. In celebrated work, Weaire and Thorpe [11] showed that short-range order is sufficient to create an optical gap. Thus, the concept of "band gap" continues to be valid even though the energy bands (i.e. $E(k)$ vs. $k$ relation) do not exist for disordered materials! Still, significant differences exist between crystalline and amorphous electronic state densities: (i) the DOS of amorphous semiconductors is essentially smooth and exhibits defect states within the gap and the 'tail' states in the vicinity of the conduction and valence band edges. In crystals, Van Hove singularities appear in the DOS in association with Brilliouin zone edges. These result in band edges delineating sharp spectral gaps. (ii) electron states near the edges tail into what would have been the forbidden gap of the crystal and (iii) a localized-delocalized transition (Anderson transition) [12-14] occurs near either band edge. The delimiter between the extended and localized states is called a "mobility edge". These three statements may be repeated mutatis mutandis for the vibrational DOS.

In this paper, we focus on the EPC in amorphous semiconductors. We discuss the electronic response to atomic motion (by treating the latter classically) and the structural response of a system to an electronic modification. In a topologically disordered semiconductor, we find that the EPC is strongly energy-dependent, and significantly larger for localized states than for extended states. This energy dependence can lead to useful anomalies in transport (e.g. high Temperature Coefficient of Resistivity (TCR) behavior in doped $a-\mathrm{Si}: \mathrm{H}$ ) and at least a partial explanation for the Meyer-Neldel rule or compensation law [15]. It also explains the strong photo-response of amorphous semiconductors and glasses (e.g. photoinduced defect creation in $a-\mathrm{Si}: \mathrm{H}$ or Staebler-Wronski effect $[16]$ ).

To put it slightly differently, we may think of the special susceptibility of the localized electron states to thermal motion of the atoms as a sort of "natural amplifier" (an amplifier that is particularly sensitive to temperature). By exploiting the unique electronic and transport properties of these states (typically by "doping into them", then measuring a derived quantity like the conductivity), devices like night-vision technologies accrue.

The lack of order also means that it is nearly impossible to construct a tractable empirical potential to model the interatomic interactions - the root of the complexity in the total energy calculations is the electronic structure, which must be grappled with in some way. This was clearly perceived in the empirical potential community in the eighties [17], but led to important advances when merged with tight-binding total energy [18, 19] or density functional methods [20]. This recognition initiated a revolution in materials theory, and enabled connections between experiment and theory previously inconceivable.

While we have reported some of these results before [21], we assemble here new insights, including simulations on different materials using high-quality plane-wave methods to eliminate any artifact associated with the in- completeness of basis sets in local-orbital-based calculations. In particular, we present new results for $a$-Se and $a$-GaN. The latter yields results different in some ways from the purely covalent materials. Turning the problem around, we will also show how the use of electronic $a$ priori information can be imposed in materials modeling as a means to carry out a program of optical engineering or "materials by design", a major current theme of condensed matter theory and simulation [22].

The rest of this paper is organized as follows. In section II, we present an elementary overview of the calculations and offer simple analytical arguments to justify some of the results. Section III discusses results for $a$-Si, $a$-Se and a-GaN, and compares and contrasts these. In section IV, we show how to obtain temperature-dependent electrical conductivity using the Kubo-Greenwood formalism, and connect this to the Meyer-Neldel rule in section V. In section VI, we show how to effectively engineer computer models to obtain desired electronic properties, and undertake a first foray into the $a b$ initio implementation of the new method. We draw conclusions in Section VII.

\section{FORMULATION}

The framework underlying these calculations is the many-body (electron and nucleon) Schrödinger equation. The first essential approximation is the BornOppenheimer or adiabatic approximation, which enables the decoupling of the electrons and phonons into separate quantum many-body problems. Still prohibitively difficult, we then make the assumption that the lattice dynamics may be treated classically. In practice, this means that the nuclei move in a potential determined by the electronic structure of the system (computed for that set of instantaneous atomic coordinates). Like others, we approach the many-electron using density functional theory in the local density approximation [23, 24]. Then, with specified initial conditions, we evolve the positions of the nuclei for a short time (of order $1 \mathrm{fs}$ ), obtaining updated coordinates and thus a new electronic Hamiltonian, with a new force field, and so the dynamics continues $^{2}$. Our final philosophical transgression is to treat the Kohn-Sham orbitals as quasiparticle states, and we take the density of electron states to just be the density of Kohn-Sham eigenvalues ${ }^{3}$.

\footnotetext{
2 Thus, in this paper, the "electronic time evolution" refers only to the instantaneous values of the electronic eigenvalues and eigenvectors for a given atomic configuration, that we have previously called "Born-Oppenheimer snapshots [25]". Naturally, the true evolution follows from a time-dependent Schrödinger equation, beyond the scope of this work [26, 27]

${ }^{3}$ Like others working in this field, we use Kohn-Sham (KS) orbitals, which have been shown to be very similar to quasiparticle states from GW calculations from Louie's group (such GW calculations provide self-energy corrections to density functional theory in the LDA). For Si, C and LiCl, Hybertsen and Louie [28]
} 
While these are substantial approximations, the resulting dynamics are reliable as seen by direct comparisons to inelastic neutron scattering measurements. These are standard assumptions used in current ab initio molecular dynamics simulations. The simulations in this paper were carried using the plane-wave code, Vienna Ab initio Simulation Package (VASP) $[29,30]$, in the local density approximation (LDA), other than briefly recounting some tight-binding work on electronic design of materials. In our earlier publications we have mostly used local orbital methods, which are computationally efficient, but with a chemically intelligent, but nevertheless, incomplete basis set. We observe that the local orbitals produced a picture very similar to what we report here, though the present results should be seen as quantitatively more reliable. ${ }^{4}$

To schematically illustrate what we are doing ${ }^{5}$, consider an orthogonal tight-binding prescription for the electronic structure and the forces. In this picture the ionic dynamics are determined from Newton's Second Law (in the form of $3 N$ coupled nonlinear ordinary differential equations):

$$
m_{\alpha} d^{2} R_{\alpha} / d t^{2}=-\frac{\partial \Phi\left(R_{1}, R_{2}, \ldots, R_{3 N}\right)}{\partial R_{\alpha}}
$$

where,

$$
\begin{aligned}
\Phi\left(R_{1}, R_{2}, \ldots, R_{3 N}\right) & =2 \sum_{i o c c} \lambda_{i}\left(R_{1}, R_{2} \ldots . R_{3 N}\right) \\
& +U\left(R_{1}, R_{2} \ldots R_{3 N}\right) .
\end{aligned}
$$

Here, $\Phi$ is the total energy, a function of the atomic coordinates $R_{\alpha}, \lambda_{i}$ are the energy eigenvalues for the tightbinding Hamiltonian $H\left(H \psi_{i}=\lambda_{i} \psi_{i}\right)$, the sum over occupied eigenvalues is the electronic contribution to the

found $99.9 \%$ overlap between GW states and the Kohn-Sham orbitals. We observed a similar state of affairs in GW calculations of quasiparticle states in Si clusters. Localized states are also artificially delocalized by LDA. On an empirical level for amorphous materials, there are many indications that it is profitable to interpret the Kohn-Sham orbitals and energies for comparisons to experiments (for example, x-ray photoemission spectroscopy (XPS) measurements on chalcogenide glasses, thermal effects in band tails of a-Si, and exciton trapping). The optical gap is always underestimated by LDA, but for most systems trends are faithfully reproduced by such an approximation. We also candidly admit that there is no other approach available, for while better theories do exist (with accurate calculations of excited states etc), they are prohibitively expensive for the materials we discuss here.

${ }^{4}$ It is well known that local-orbital Hamiltonians generally do well in these systems, sometimes being closer to experiment than plane waves. This could be due to compensating errors (for example the tendency of the LDA to produce overly delocalized states and a localized basis set which is incomplete and therefore yields states more localized than a complete-basis Kohn-Sham calculation.

5 The results reported in this paper are based on plane-wave DFT unless otherwise indicated. This discussion is offered to clarify the approach without all the mathematical detail. total energy (the so-called band-structure energy), and $U$ is a short-range repulsive potential between ions. The greatest complexity emerges in Eq. 2, for which the computation of the energy eigenvalues $\lambda$ requires a matrix diagonalization, so that the dependence of $\Phi$ on $R_{\alpha}$ may take a highly complex, non-local and non-analytic functional form. Equation 1 represents the influence of the electrons on the particle coordinates in the form of a second-order differential equation and Eq. 2 identifies the functional dependence of the total energy in terms of the electronic structure.

Equations 1 and 2 highlight the role of the HellmannFeynman derivatives,

$\nabla_{\alpha} \lambda_{i}=\frac{\partial \lambda_{i}\left(R_{1}, R_{2}, \ldots, R_{3 N}\right)}{\partial R_{\alpha}}=\left\langle\psi_{i}\left|\frac{\partial H\left(R_{1}, R_{2}, \ldots, R_{3 N}\right)}{\partial R_{\alpha}}\right| \psi_{i}\right\rangle$,

which are gradients of the electronic eigenvalues. This result is particularly useful because the RHS is easily computed and necessary for any molecular dynamics simulation based on an interatomic potential derived from electronic structure. Since they are gradients, they represent the direction of the most rapid increase of the eigenvalues in the configuration space. These gradients have special utility for modeling of photoresponse [31] and computational gap engineering [32].

\section{A. Lattice dynamics and electronic fluctuations}

To appreciate the difference in electronic (and optical) properties between crystals and semiconductors, we begin by addressing the time evolution of the Kohn-Sham (KS) eigenvalues from molecular-dynamics simulations as shown in Fig. 1. Here we show the fluctuations of the KS eigenvalues at room temperature $(300 \mathrm{~K})$ in crystalline $\mathrm{Si}(c-\mathrm{Si})$ and amorphous $\mathrm{Si}(a-\mathrm{Si})$. The thermal fluctuations of the KS eigenvalues are considerably larger for the amorphous system than for the crystal, and the degree of fluctuation is obviously energy-dependent. It's also apparent that the tail states have a large fluctuation that 'fills in' much of the gap region ${ }^{6}$. The energy scale of the problem is the thermal energy $k_{B} T$, of the order of 0.025 $\mathrm{eV}$, at $\mathrm{T}=300 \mathrm{~K}$. While this value roughly characterizes the magnitude of fluctuations observed for the crystal,

\footnotetext{
${ }^{6}$ A nearly universal approximation for semiconductor models with more than 100 atoms is the $\Gamma$-point approximation. Electron states are computed only at $\mathbf{k}=\mathbf{0}$, from which total energies and forces are computed based upon the pious hope that the bands are flat (with respect to $\mathbf{k}$ dispersion) for such a large cell. In a similar way, the density of states is usually taken at $\Gamma$ only. It is noteworthy that for the 216-atom crystal cell there is a "gap" between -6.8 and $-6.4 \mathrm{eV}$ (Fig. 1), appearing only because we did not integrate over the Brillouin zone of the cell (that is, we sampled only the $\Gamma$ point), a sobering reminder of how slowly we approach the thermodynamic limit from an electronic perspective [33]! Through the rest of the paper, we employ only $\Gamma$.
} 
the thermal excursions of the Kohn-Sham states may be significantly larger (more than a factor of 10) near the Fermi level. Further, the fluctuations decay in amplitude as we move away from the conduction or valence band edges (far from the Fermi level) to a region where the states are normally delocalized. This leads to the conclusion that localization of a given eigenstate amplifies the fluctuations for the eigenvalue associated with that state. We have separately observed that eigenfunctions conjugate to localized eigenvalues change dramatically in response to thermal motion of atomic positions [34].

A universal feature of disordered systems is the characteristic presence of band-tail states with an exponential form (of the density of states) observed in diverse experiments [35]. As the preceding paragraph suggests, these tail states are temperature dependent. Ab initio calculations on static $a$-Si models suggest that the nature of the tail states are intimately connected with the degree of bond-length disorder in the network [36]. In particular, they exhibit a filamentary character in real space, which is associated with Si sites with short bond lengths near the valence edge and long bond lengths near the conduction edge. The temperature dependence of these tails has been observed in photoemission experiments [37], and a simple theory of this has been given elsewhere $[21,38]$.

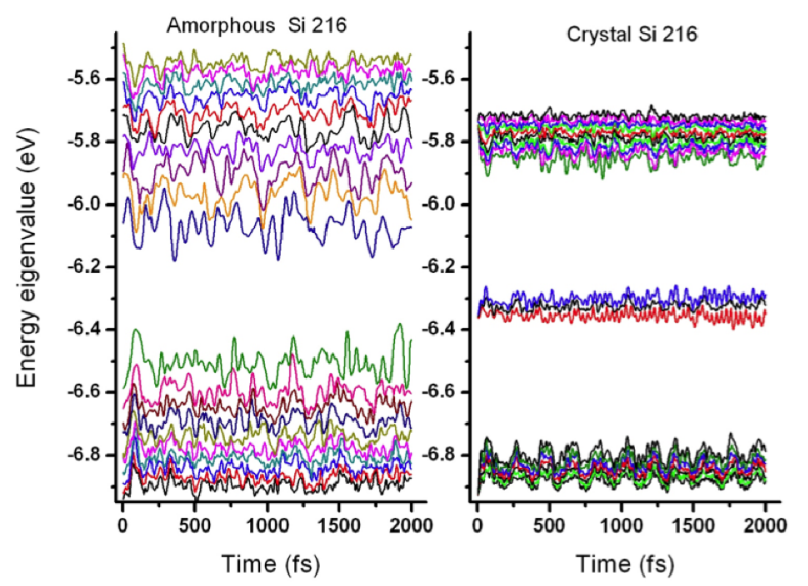

FIG. 1: Thermal fluctuations of Kohn-Sham eigenvalues in the vicinity of the Fermi level (near $-6.3 \mathrm{eV}$ ) for a 216-atom model of $a_{-} \mathrm{Si}$ (left) and $c-S i$ (right), both at $300 \mathrm{~K}$. The natural energy scale for the lattice in equilibrium is $k_{B} T \approx 0.025 \mathrm{eV}$, not far from what is seen for c-Si. The fluctuations in $a_{-} \mathrm{Si}$ near the Fermi level can be ten times this value.

\section{B. Electron-phonon coupling, eigenvalue fluctuations and localization}

In earlier works $[21,25,39,40]$, we obtained simple estimates of the strength of the EPC (for $a-\mathrm{Si}$ ) by plotting the variance of the thermal fluctuation of the KohnSham eigenvalues versus the localization, gauged by In- verse Participation Ratio (IPR). ${ }^{7}$ But before doing so, we briefly describe the origin of the electron-phonon interaction in solids based on simple physical reasoning.

The first order contribution to the electron-phonon interaction originates from the off-diagonal elements of the non-adiabatic coupling operator that links the electronic eigenvalues to the various atomic degrees of freedom due to the movement of the atoms in a thermally-vibrating lattice. This follows from the BornOppenheimer ansatz ${ }^{8}$ : the total wave function of a solid can be written as a linear combination of the product of the electronic eigenfunctions (for a fixed atomic configuration) with expansion coefficients that depend on the instantaneous position of the ions. Following Bloch [41], an approximate expression for the electron-phonon interaction can be obtained by estimating the deformation potential of the ions. Owing to the movement of the atoms in a vibrating lattice, an electron at $\mathbf{r}_{k}$ sees a change in the ionic potential $\delta V^{e, i}\left(\mathbf{r}_{k}, \mathbf{R}_{l}\right)$ as the atom moves from its equilibrium position at $\mathbf{R}_{l}^{0}$ to $\mathbf{R}_{l}$. For a small displacement, the interaction may be taken to be proportional to the displacement $\delta \mathbf{R}_{l}^{0}$ of the ion, i.e.

$$
\begin{aligned}
V^{e, i}\left(\mathbf{r}_{k}, \mathbf{R}_{l}\right) & =V^{e, i}\left(\mathbf{r}_{k}, \mathbf{R}_{l}^{0}+\delta \mathbf{R}_{l}^{0}\right) \\
& \approx V^{e, i}\left(\mathbf{r}_{k}, \mathbf{R}_{l}^{0}\right)+\delta \mathbf{R}_{l}^{0}\left(\frac{\delta V^{e, i}}{\delta \mathbf{R}_{l}}\right)_{\mathbf{R}_{l}=\mathbf{R}_{l}^{0}}
\end{aligned}
$$

In the above equation, the first term indicates the equilibrium value of the electron-ion potential at $\mathbf{R}_{l}^{0}$. Thus the total additional potential experienced by an electron at site $r_{k}$ is given by the sum of the contribution from all atomic sites,

$$
\delta V^{e, i}\left(\mathbf{r}_{k},\left\{\mathbf{R}_{l}\right\}\right)=\sum_{l} \delta \mathbf{R}_{l}^{0}\left(\frac{\delta V^{e, i}}{\delta \mathbf{R}_{l}}\right)_{\mathbf{R}_{l}=\mathbf{R}_{l}^{0}}
$$

Assuming that the total electron-ion interaction in a solid can be expressed as a pairwise sum over individual electrons, a simple expression for the total deforming potential can be written as,

$$
\delta \mathbf{V}^{e, i}=\sum_{k} \delta V^{e, i}\left(\mathbf{r}_{k},\left\{\mathbf{R}_{l}\right\}\right)=\sum_{k, l} \delta \mathbf{R}_{l}^{0}\left(\frac{\delta V^{e, i}}{\delta \mathbf{R}_{l}}\right)_{\mathbf{R}_{l}=\mathbf{R}_{i}^{0}}
$$

7 The latter provides an estimate for the degree of localization of a wave function. A large value of IPR reflects a spatially compact or localized state, whereas a small value indicates an extended state. For convenience, we summarize the argument here.

8 The adiabatic approximation is obtained by neglecting the off-diagonal elements of the non-adiabatic coupling operator, whereas the Born-Oppenheimer approximation ignores both the diagonal and off-diagonal elements. Electron-phonon coupling originates from the small but finite off-diagonal elements, and thus can be treated as a perturbation for a small displacement of ions. 
In a thermally-vibrating lattice $\mathbf{R}_{l}$ can be written as $\mathbf{R}_{l}=\mathbf{R}_{l}^{0}+u\left(\mathbf{R}_{l}^{0}\right)$, where $u\left(\mathbf{R}_{l}^{0}\right)$ is the vibrational displacement at site $\mathbf{R}_{l}^{0}$, to obtain the electron-lattice interaction,

$$
\mathbf{H}_{e, p}=\sum_{k, l} u\left(\mathbf{R}_{l}^{0}\right)\left(\frac{\delta V^{e, i}\left(\mathbf{r}_{k},\left\{\mathbf{R}_{l}\right\}\right.}{\delta u\left(\mathbf{R}_{l}^{0}\right)}\right) .
$$

Equation 6 can be used to study the electron-phonon interaction in solids by expressing $u\left(\mathbf{R}_{l}^{0}\right)$ and $\frac{\partial V}{\partial u(\mathbf{R})}$ in second quantization forms in terms of phonon and electron creation/annihilation operators, respectively.

We now proceed to establish a connection between electron-phonon coupling and wave-function localization for the electrons. Consider an electronic eigenvalue $\lambda_{n}$ near the band gap. The sensitivity of $\lambda_{n}$ due to an arbitrary small displacement of an atom (possibly thermally induced) can be estimated using the Hellmann-Feynman theorem [42],

$$
\frac{\partial \lambda_{n}}{\partial \mathbf{R}_{\alpha}}=\left\langle\psi_{n}\left|\frac{\partial \mathbf{H}}{\partial \mathbf{R}_{\alpha}}\right| \psi_{n}\right\rangle
$$

Here we have assumed that the basis functions are fixed and $\left|\psi_{n}\right\rangle$ are the eigenvectors of the Hamiltonian $\mathbf{H}$. For small lattice distortion $\left\{\delta \mathbf{R}_{\alpha}\right\}$, the corresponding total change in $\delta \lambda_{n}$ is,

$$
\delta \lambda_{n} \approx \sum_{\alpha=1}^{3 N}\left\langle\psi_{n}\left|\frac{\partial \mathbf{H}}{\partial \mathbf{R}_{\alpha}}\right| \psi_{n}\right\rangle \delta \mathbf{R}_{\alpha}
$$

where $\mathrm{N}$ is the total number of atoms in the model. In harmonic approximation,

$$
\delta \mathbf{R}_{\alpha}=u\left(\mathbf{R}_{\alpha}\right)=\sum_{\omega}^{3 N} A(T, \omega) \cos \left(\omega t+\phi_{\omega}\right) \chi_{\alpha}(\omega),
$$

where $\chi_{\alpha}(\omega)$ is the normal mode with angular frequency $\omega, A$ is the temperature-dependent amplitude, and $\phi_{\omega}$ is an arbitrary phase angle. By defining

$$
\Xi_{n}(\omega)=\sum_{\alpha=1}^{3 N}\left\langle\psi_{n}\left|\frac{\partial \mathbf{H}}{\partial \mathbf{R}_{\alpha}}\right| \psi_{n}\right\rangle \chi_{\alpha}(\omega),
$$

we compute $\left\langle\delta \lambda_{n}^{2}\right\rangle$ as

$$
\left\langle\delta \lambda_{n}^{2}\right\rangle=\lim _{\tau \rightarrow \infty} \frac{1}{\tau} \int_{0}^{\tau} d t \delta \lambda_{n}^{2} \approx\left(\frac{k_{B} T}{2 M}\right) \sum_{\omega \neq 0}^{3 N} \frac{\Xi_{n}^{2}(\omega)}{\omega^{2}}
$$

where $\Xi_{n}(\omega)$ can be interpreted as electron-phonon coupling function between electron $n$ and phonon $\omega$.

It may be noted that Eq. 7 includes the contribution from the electron-phonon term in Eq. 6 and possibly contribution from additional degrees of freedom depending on the approximations involved in the electronic structure calculations. Thus, our definition of the electronphonon coupling in Eq.9 is consistent with the Bloch form in Eq. 6. By neglecting various canceling contributions, it is not difficult to show that the electron-phonon coupling $\Xi_{n}(\omega)$ between electron/band $n$ and phonon $\omega$ satisfies [40]:

$$
\Xi_{n}^{2}(\omega) \sim \mathcal{I}_{n}
$$

Furthermore, within this simplified framework:

$$
\left\langle\delta \lambda_{n}^{2}\right\rangle \propto \mathcal{I}_{n}
$$

We forthrightly emphasize that these arguments are very crude, though it is remarkable that there exists a direct proportionality between a static property (IPR) and the RMS thermal fluctuation of energy eigenvalues associated with localized eigenstates (as clearly seen in simulations of $a-\mathrm{Si}$ ).

Here, the $\mathrm{IPR}, \mathcal{I}$ is computed as:

$$
\mathcal{I}=\frac{\sum_{i} a_{i}^{4}}{\left(\sum_{i} a_{i}^{2}\right)^{2}}
$$

In the preceding, the $a_{i}$ are components of the eigenvector being analyzed, projected onto atomic s, p and d states as provided by VASP.

In the course of analyzing new simulations for this manuscript, we noted that a more general (closer) linkage between localization $\mathcal{I}$ and thermal variation of eigenvalues can be stated in the form of:

$$
\delta \lambda_{r m s}=\alpha \overline{\mathcal{I}}+\beta
$$

and $\delta \lambda_{r m s}=\sqrt{\bar{\lambda}^{2}-(\bar{\lambda})^{2}}$. In the preceding, the raised bar means average over the simulation at constant temperature $T$. $\alpha, \beta$ are constants that depend on the type of material. The value of $\alpha$ gives the 'amplification factor', a measure of how strongly the lattice vibrations affect electronic energies of localized states. One may suppose that parameter $\alpha$ is a partial indicator of the TCR of a materials doped into the fluctuating states.

\section{Structural change from electronic/optical modification}

Topological or chemical irregularities in amorphous networks lead to localized electron states in the gap or in the band tails. If such a system is exposed to light of appropriate wave length, then it becomes possible for the light to induce transitions of electrons from the top of the occupied states to low-lying unoccupied (conduction) states. For the present paper, we will not concern ourselves with the subtleties of how the EM field introduces the transition; we will simply assume that a photo-induced promotion of an electron occurs, by depleting the occupied states of one electron forming a hole 
and moving the electron to the bottom of the unoccupied conduction states. Such a process is explicitly nonequilibrium and the response suffered by the system is of key interest. This results in a change of the HellmannFeynman forces leading to structural rearrangements, either negligible or significant, depending on the flexibility or stability of the network, localization of the states and EPC. The timescale associated with this relaxation from occupation-induced changes are short (e.g. a few phonon periods), which can be exploited to develop novel applications in solids involving ultrafast processes [43]. This approach was pioneered by Peter Fedders and coworkers [44].

Recently, in a series of articles, we have reported structural changes associated with light irradiation in $a-\mathrm{Si}$, $a$-Se, and $\mathrm{a}_{-} \mathrm{As}_{2} \mathrm{Se}_{3}[31,45-47]$. We will not dwell on the system-specific details here but instead describe the short-time response of such systems suffering an occupation change of electronic states. In particular, we address the case of structural changes from placing an electron at the bottom of the conduction edge. We discuss this for $a-\mathrm{Si}$ and $a-\mathrm{Se}$ in this paper.

\section{RESULTS FOR AMORPHOUS PHASES OF SI, SE AND GAN}

In the following, we present results from molecular dynamics simulations of $a-\mathrm{Si}, a-\mathrm{Se}$, and $a$ - $\mathrm{GaN}$ to link the thermal variation of Kohn-Sham eigenvalues to temperatures and static properties of the eigenfunctions like Inverse Participation Ratio (IPR). The first two materials are covalent, and the third is significantly ionic. Amorphous selenium has the feature that the states near the Fermi level are built from $p$-orbitals with associated phenomena like valence alternation $[31,48,49]$. Amorphous silicon has band tails that originate from linear combinations of $s$ and $p$ states of bonding and anti-bonding form. On the other hand, $a-\mathrm{GaN}$ is a partly ionic semiconductor in which the valence and conduction states involve quite different hybridizations, with the conduction tail consisting primarily of mixtures of both $\mathrm{Ga}$ and $\mathrm{N} s$ states. The conduction states of $a-\mathrm{GaN}$ are less localized than the valence states, presumably because these states exhibit little dependence on local bond-angle disorder because of the isotropy of the $s$-orbitals from which the eigenstates are built. Surprisingly, a-GaN also displays an essentially extended midgap state.

\section{A. Amorphous Silicon}

Here we employ a realistic 216 -atom continuous random network model of $a$-Si due to Djordjevic et al. [50, 51]. The model, commonly referred to as the 'WWW model', is discussed extensively elsewhere, and reproduces the properties of a-Si well for a small model.

In Fig. 2 we provide the electronic density of states

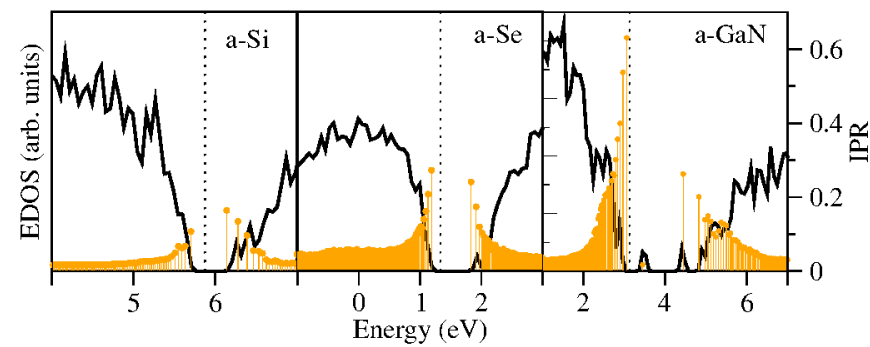

FIG. 2: Electronic Density of States (EDOS) and Inverse Participation Ratio (IPR) for $a$-Si, $a$-Se and $a$-GaN models discussed in this paper. The black curves represent the EDOS and the orange drop lines represent IPR. The dotted lines represent the position of Fermi level for each model. The models being represented in the figure are described in the sections to follow.

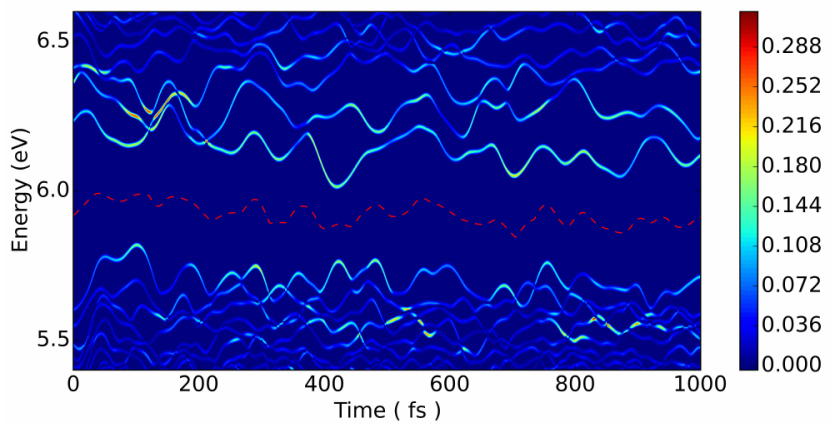

FIG. 3: Thermal fluctuations of Kohn-Sham eigenvalues near the gap in $a-\mathrm{Si}$. The eigenvalues are adiabatically evolving at a constant temperature of $300 \mathrm{~K}$. The colors represent the localization of the states as measured by IPR (Eq. 13). The dotted line in red represents the Fermi Energy.

(EDOS) for $a-\mathrm{Si}$ along with the EDOS of $a$-Se and $a-\mathrm{GaN}$. The IPR is slightly larger on the conduction tail than on the valence tail. We begin by presenting results for thermally induced fluctuations of the Kohn-Sham states. In Fig. 3 we show the thermally induced fluctuation in the eigenvalues with color indicating extent of localization. The fluctuation for the eigenvalues in the gap are quite dramatic, varying over tenths of an $\mathrm{eV}$ for the lowest conduction states.

Beside the variation in energy of electronic states, the thermal motion induces fluctuation in localization of these states as well. We note from Fig. 3, that such fluctuation in localization is higher for states with commensurate high fluctuation in energy (i.e. the band-edge states). We find that thermal fluctuation modulates the bond lengths and bond angles, resulting in a reshuffling of the atomic contributions to electronic states. If these states are already localized, such reshuffling can induce dramatic changes to the extent of localization. Inspection of Fig. 3 suggests that at a time near $100 \mathrm{fs}$, the conduction tail states become momentarily very localized. As an illustrative tale of variation in electronic structure induced by thermal disorder, we find that at this 


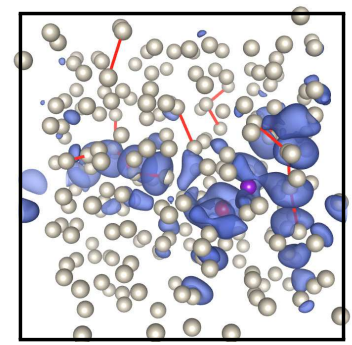

FIG. 4: The combined charge density of lower three conduction states when these states are most localized. This image corresponds to a snapshot at time $=100 \mathrm{fs}$ in figure 3 . The figure shows these states are most localized around the defect atoms (represented in color, purple: 5-fold, red: 3-fold) and around long bonds (bonds longer than $2.5 \AA$ are shown as red sticks in the figure).

instant, the states are localized around long bonds or broken bonds. We find that a pair of short-lived defects consisting of one dangling bond (a three-fold bond) and one floating bond (a five-fold bond) that are adjacent to each other are formed around this time. In Fig. 4, we show the charge density of the three lowest conduction tail states for this particular short-lived conformation. We note that these three states are largely localized around the newly formed defect pair, and also around long bonds in the network. Fig. 4 shows the bonds longer than $2.5 \AA$ and its evident that these conduction tail states have large projections onto these sites. Note that the long bonds in the cell show a spatial correlation among themselves forming a filament like pattern $[36,52]$. It is notable that this defect is thermally induced and transient. After $200 \mathrm{fs}$, we see short-lived surges in the extent of localization of tail states and these surges correspond to various thermally induced defects and bond length fluctuations.

In Fig. 5 we see that there is a linear correlation between the RMS fluctuation of the eigenvalues and the the IPR (localization), confirming Equation 14. In Fig. 6 , we observe that the band tail states fluctuate far more than the deep states for a given temperature. Fig. 6 also portrays the temperature dependence of the fluctuation of eigenvalues across the spectrum. We observe that temperature amplifies $\delta \lambda_{n}$ and the amplification is significantly higher for the band edge states.

In $a-\mathrm{Si}: \mathrm{H}$, there is an extensively studied process of light-induced photodegradation: the Staebler-Wronski effect, in which light exposure leads to the creation of charge carrier traps, presumably from defects created by the light exposure. Fedders and coworkers [44] have studied this problem atomistically by creating creating an electron-hole pair and tracking the creation and annihilation of structural defects ${ }^{9}$. In the spirit of their

\footnotetext{
9 The scheme can be understood to be a sophisticated local heating approach, with the feature that the intensity of the local
}

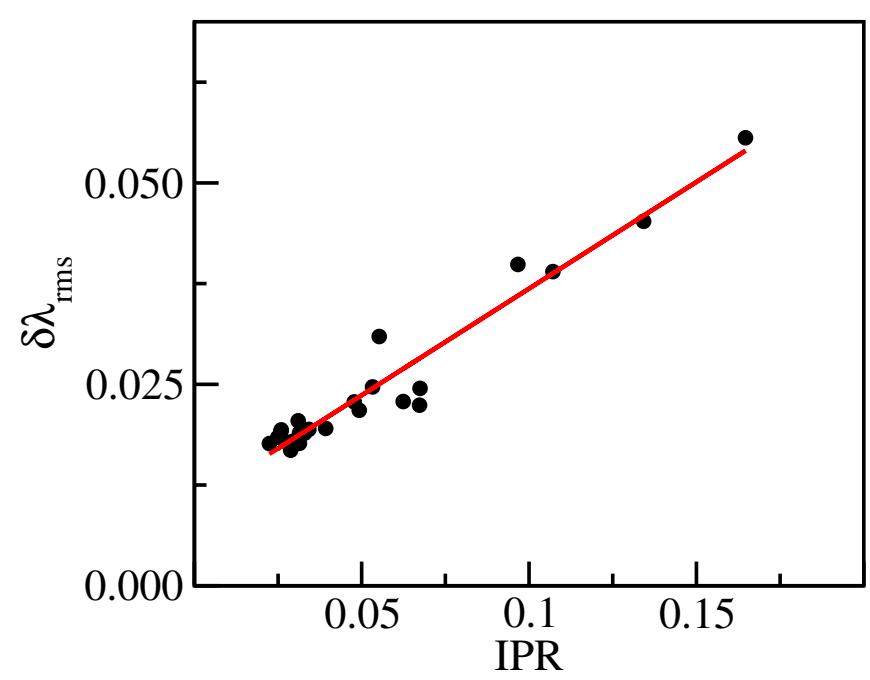

FIG. 5: The correlation between RMS fluctuation of eigenvalues around the gap and corresponding inverse participation ratio (IPR) at $300 \mathrm{~K}$ for the electronic ground state in a-Si (Eq. 14). The bold red line is the linear fit of the plotted values. The fitted value of $\alpha$ (Eq. 14) for a linear fit is 0.26 .

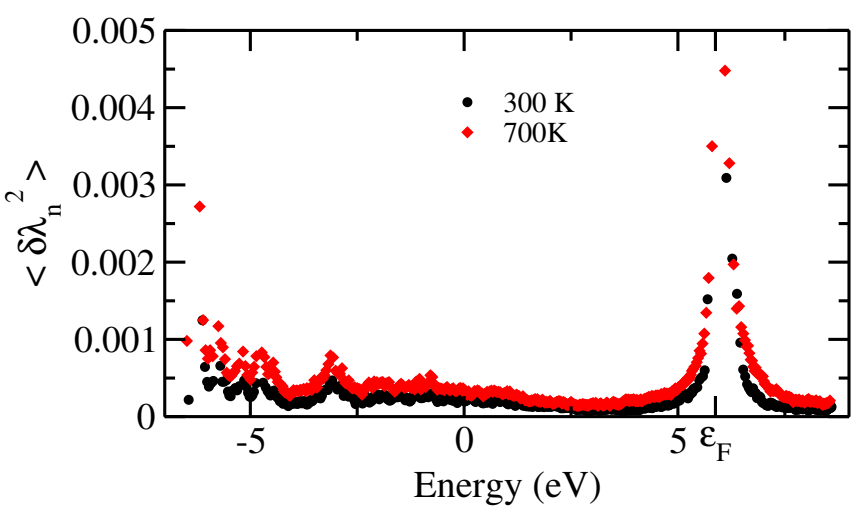

FIG. 6: Temperature dependence of mean squared fluctuation of eigenvalues. The fluctuation is calculated over constant temperature $\mathrm{MD}$ at $300 \mathrm{~K}$ and $700 \mathrm{~K}$. Symbol $\varepsilon_{F}$ represents the position of Fermi energy. Note that the band edge states fluctuate more than the deep states. Also, the temperature modulation of the energy is higher for the tail states than for the deeper states.

approach, here we have have conducted constant temperature MD using VASP [29, 30] and promoted an electron to the bottom of the conduction states. The LUMO drops precipitously into the gap, nearly closing the gap momentarily, near $t=600 \mathrm{fs}$ (see Fig 7). This change is accompanied by creation and annihilation of short-lived geometrical defects in the network. We have empirically

heating is critically determined by the electron-phonon coupling and the Hellmann-Feynman forces (Eq. 3) associated with the occupation change. 


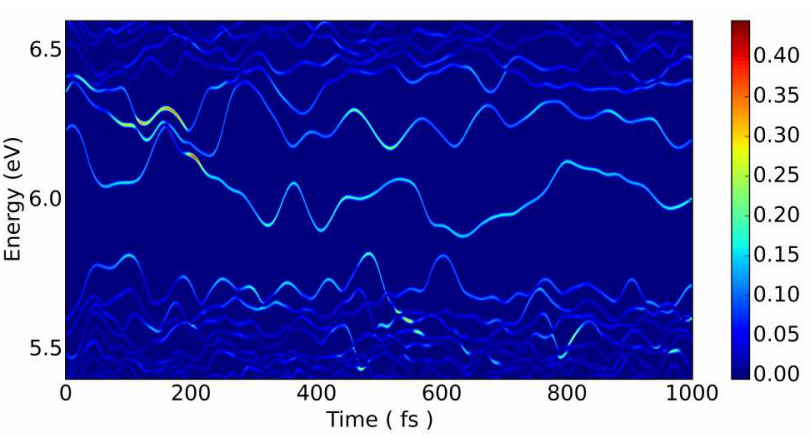

FIG. 7: Thermal fluctuations of Kohn-Sham eigenvalues near the gap in excited $a-\mathrm{Si}$. The eigenvalues are evolving under constant temperature at $300 \mathrm{~K}$ when an electron is promoted to conduction band. The color represents the localization of the states as measured by Inverse participation ratio (IPR). Note that this is a non-equilibrium simulation as the system responds to the promotion of an electron to the bottom of the conduction states. Fermi level for the unexcited system at $\mathrm{t}=0$ is at $5.88 \mathrm{eV}$.

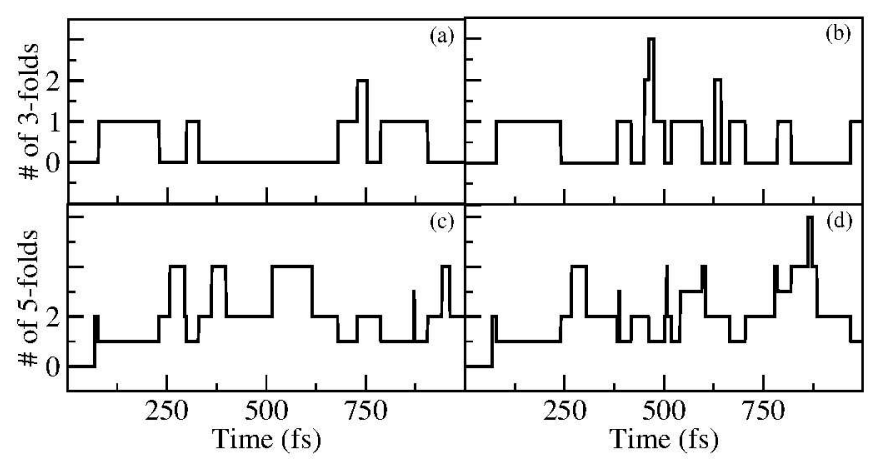

FIG. 8: Thermally induced geometrical defects in excited and unexcited $a_{-}$Si. (a) and (c) are the evolution of defects under constant temperature MD at $300 \mathrm{~K}$ in unexcited 216 -atom $a$ Si. (b) and (d) are those for an excited system. Coordination is defined by a radius $r_{c}=2.7 \AA$

observed that the number of geometrical defects tend to increase as a result of promotion of an electron in a conduction state. Figure 8 shows the thermal fluctuation of geometrical defects at $300 \mathrm{~K}$ for the time interval that corresponds to the energy fluctuations in Fig 7. A plausible explanation of this observation is that the lowest conduction state is already localized around coordination defects and long bonds. The occupation of a conduction state with an electron causes these atoms to experience the Hellmann-Feynman force coming from the newly occupied state and hence these atoms become much more active. We have observed that the states in an excited system are more localized than those in the corresponding unexcited system.

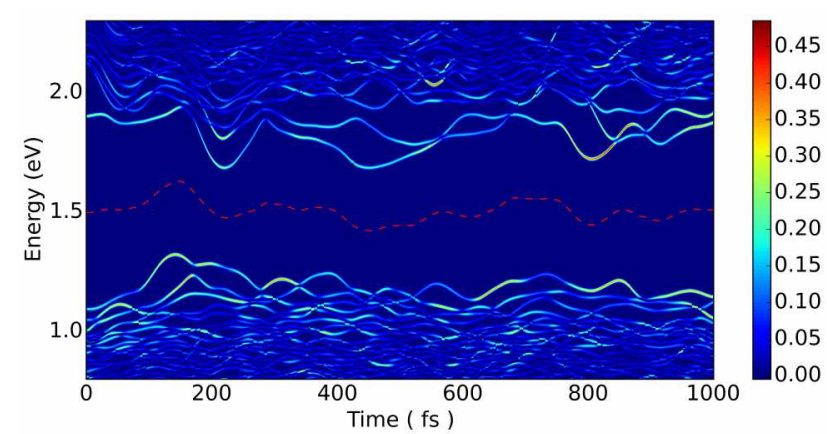

FIG. 9: Thermal fluctuations of Kohn-Sham eigenvalues near the gap in $a$-Se. The color dimension corresponds to the localization of the states as measured by (IPR). The dotted line in red represents Fermi Energy.

\section{B. Amorphous Selenium}

Amorphous selenium is different from $a-\mathrm{Si}$ in that its network is less constrained, which may be expected to play out in the EPC of localized states. We make use of a 216-atom model of Zhang and Drabold [31] and relax it using LDA in a plane wave basis. The model represents the material reasonably well as reported in earlier work $[31,43]$. It is notable from Fig. 2 that the density of states is higher near the Fermi level for $a$-Se. The localization of the tail states is fairly similar at either band edge of the optical gap.

We perform constant temperature dynamics at $300 \mathrm{~K}$ using a Nosé thermostat and monitor the evolution of Kohn-Sham eigenvalues. In Fig. 9 we show the thermal fluctuation of energy eigenvalues near/inside the band edges. We see that the edge states fluctuate substantially more than the extended states (well into the valence and conduction bands) and they are more localized compared to the deep states. The system has an under coordinated and an over coordinated atoms to begin with. The equilibrium edge states are usually strongly localized around these imperfections. The dynamics introduces many short-lived defect states in the system.

In Fig. 10 we examine the correlation between the RMS variation of the eigenvalues and the thermally averaged IPR as in Eq. 14. Here, we see a strong correlation between the fluctuation of energy and the spatial localization of the state. We observe separate correlation for conduction and valence states. In relation to Eq. 14, we note that the 'amplification' factor $\alpha$ for the conduction states is twice as big as for valence states. Figure 11 indicates once again that there is connection between localization and electronic response to phonons. We note, as in Fig 6, that the fluctuation of localized tail states is significantly higher than that of the extended valence and conduction states.

In Fig 12, we consider the nonequilibrium (adiabatic) time development of the system - the response to adding a carrier to the LUMO state. We note that the LUMO plunges nearly $1 \mathrm{eV}$ across the gap in a time of about 400 


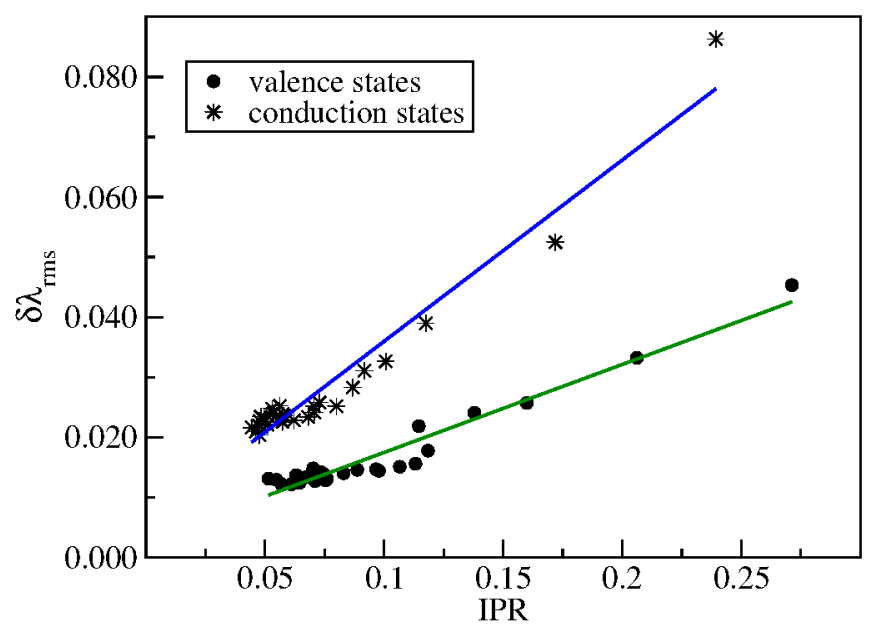

FIG. 10: The correlation between RMS fluctuation of eigenvalues around the gap and corresponding inverse participation ratio (IPR) in $a$-Se (Eq. 14). The fluctuation is calculated over constant temperature MD at $300 \mathrm{~K}$. Note that the fluctuation is higher for conduction states than for valence states. The correlation breaks neatly into two "branches", one for valence and one for conduction tail states. The green and blue lines represent a linear fit of the plotted values. The fitted values of $\alpha$ for the linear fits are 0.15 for valence states and 0.30 for conduction states.

fs (see Fig 13 to follow the evolution of LUMO on longer timescale). Whenever an electron fills a conduction state, the system tries to lower the total energy of the system, typically by the relevant state dropping into the gap, thereby reducing its (electronic) energy, and there will be geometrical changes associated with this electronic drop - bond breaking and/or formation. Some systems like Si may be too constrained to allow geometrical rearrangements enabling such an electronic plunge. Amorphous Se, on the other hand, has a highly flexible (essentially polymeric) structure and hence can provide for the lowering of the newly filled conduction state. This suggests an explanation for why the photo-darkening effect in $a$-Se is so pronounced (see figure 13).

\section{Amorphous Gallium Nitride}

The case of the partly ionic system $a$-GaN makes an interesting counterpoint to the purely covalent materials described in earlier sections. The first peculiarity of this material is that the valence tail is more localized than the conduction tail. The reason for this is that the valence tail is susceptible to bond angle disorder whereas the conduction tail is predominantly sensitive to bond length disorder (which is comparatively mild) [53]. In Fig. 14 we note that the valence states are densely packed together with notable fluctuations right at the band edge. Remarkably, a-GaN displays an essentially extended midgap (deep acceptor) state (see Fig 2). This is of significant interest, since such states are unknown in covalent systems

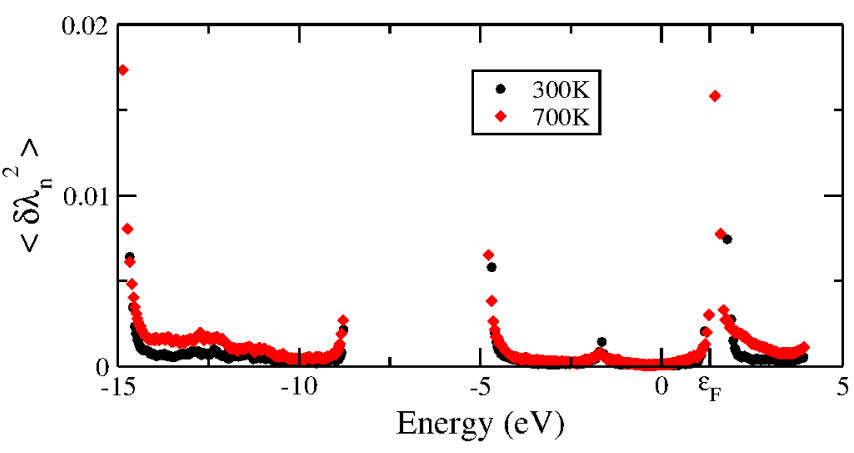

FIG. 11: Temperature dependence of mean squared fluctuation of eigen values in $a-\mathrm{Se}$. The fluctuation is calculated over constant temperature MD at $300 \mathrm{~K}$. and $700 \mathrm{~K}$. Symbol $\varepsilon_{F}$ represents the position of Fermi energy. Note, in addition to the observations in Fig. 6, that deep states are virtually unaffected by temperature whereas the edge states see strong modulation.

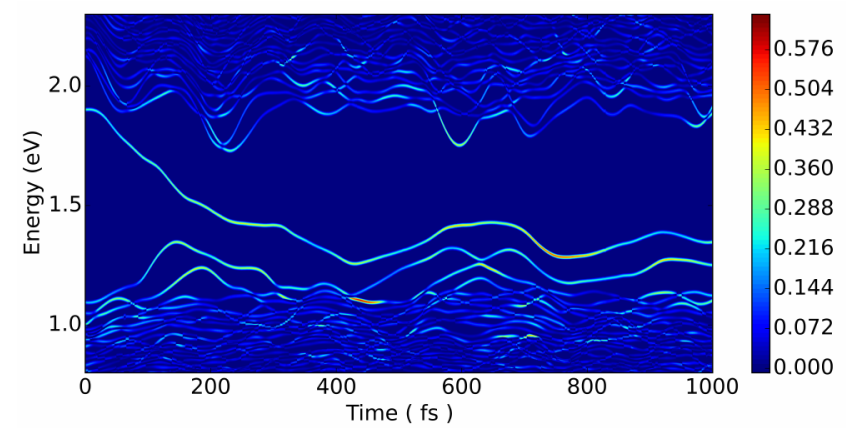

FIG. 12: Thermal fluctuations of Kohn-Sham eigenvalues near the gap in excited $a$-Se. The system is kept at T=300 K when an electron is promoted to conduction band. The color dimension maps to the localization of the states (IPR). Fermi level for the unexcited system at $\mathrm{t}=0$ is at $1.33 \mathrm{eV}$.

like those discussed earlier. As we have suggested elsewhere, these distinctive extended states must yield better electronic conduction than in covalent systems [53, 54].

What is also of interest is the meandering conduction state (starting at near $4.5 \mathrm{eV}$ at $\mathrm{t}=0$ ), which shows a thermal dispersion of nearly $1 \mathrm{eV}$, the largest we have noted in any of these systems. In this case, we see a first example contradicting the general rule "more localized implies more fluctuation", clearly seen in Fig. 15. The linear correlation is remarkably well maintained, but again split into different branches for valence and conduction edges. ${ }^{10}$ Unlike covalent systems studied earlier, the

10 Within a given band - conduction or valence, the rule of linear correlation between IPR and RMS fluctuation is preserved. The partitioning into two branches isn't surprising since hybridization of the eigenstates near the two band edges is quite different for GaN, suggesting that in general the two edges need to be treated separately. 


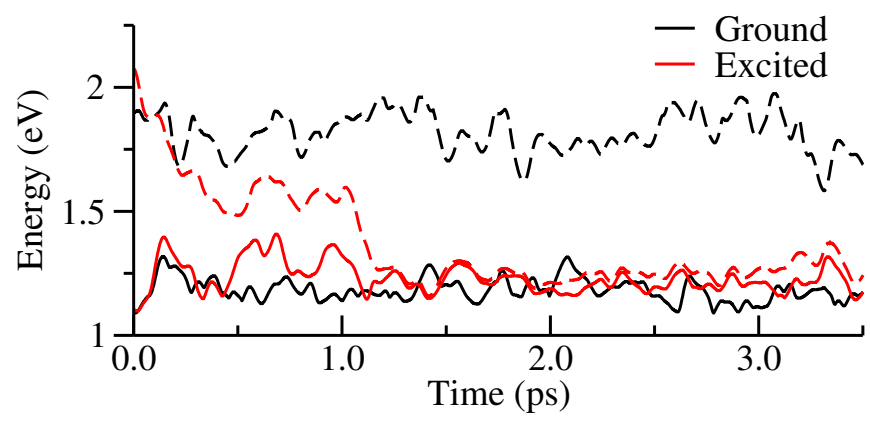

FIG. 13: The evolution of HOMO and LUMO states of $a$-Se. The ground state represents the constant temperature MD at $300 \mathrm{~K}$ whereas the excited state refers to similar MD run when an electron is promoted to conduction band.

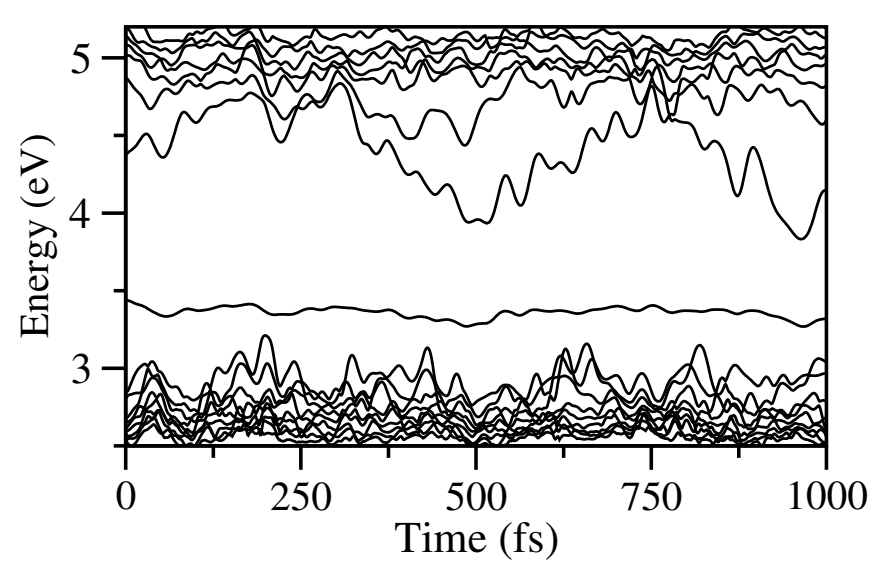

FIG. 14: Thermal fluctuations of Kohn-Sham eigenvalues near the gap in GaN. The eigenvalues are evolving under constant temperature at $300 \mathrm{~K}$. Note the energetically itinerant conduction state level near $4.5 \mathrm{eV}$ at $t=0$ and the extended gap state near $3.5 \mathrm{eV}$ at $t=0$. The Fermi level at $\mathrm{t}=0$ is at $3.13 \mathrm{eV}$

state near $3.5 \mathrm{eV}$ at $\mathrm{t}=0$ is extremely extended despite being nearly a "midgap" state. As expected from the discussion of section II B, such a delocalized state has a very weak EPC and barely shows thermal modulation at all.

In Fig. 15, the strong linear correlation between IPR and fluctuation is again observed. The state near $4.5 \mathrm{eV}$ at $t=0$ of Fig. 14 shows very high fluctuation compared to the rest of the conduction states and is not included in the plot. There are two distinct linear plots in Fig. 15, - one for the valence states and the other for the conduction states. The thermally-averaged values of IPR and RMS variation are plotted along the abscissa and ordinate, respectively. Again, we plot it in this way because it shows a better correlation than the result implied by Eq. 12. This is an empirical, but evidently strong, correlation. It may be noted that, for all three cases, the intercept $\beta$ (from Eq. 14) provides an estimate for the fluctuation of an ideally extended state $(\mathcal{I}=0)$, which is of the order of $k_{B} T$ as expected at $\mathrm{T} \mathrm{K}$.

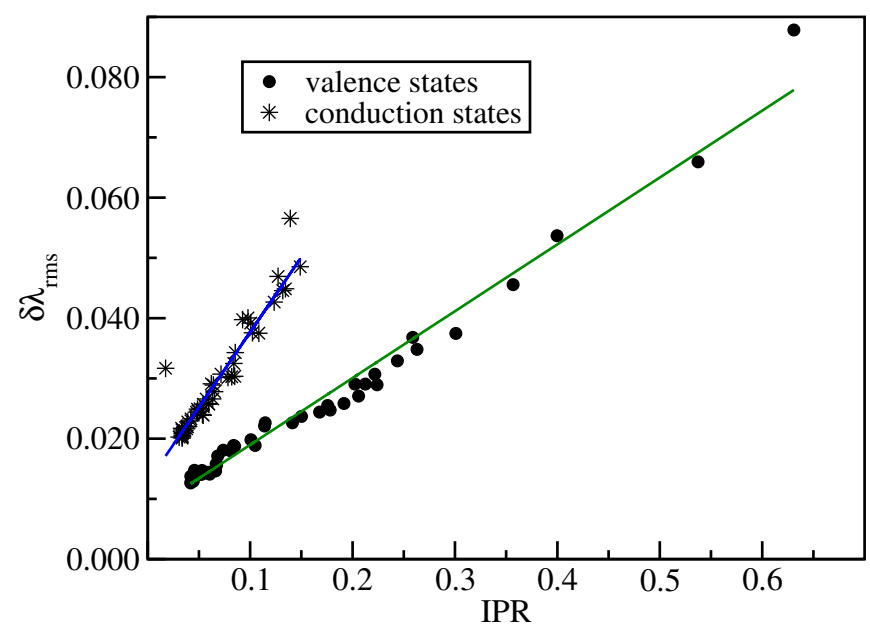

FIG. 15: The correlation of RMS fluctuation of eigenvalues around the gap and corresponding inverse participation ratio (IPR) in GaN, as described by Eq. 14. The fluctuation is calculated over constant temperature MD at $300 \mathrm{~K}$. Note that the fluctuation is higher for conduction states than for valence states. As was the case in Se, the correlation is observed in two separate branches for the conduction and valence edge states. The green and blue lines represent the linear fit of the plotted values. The observed value of $\alpha$ for the linear fits are 0.11 for valence states and 0.25 for conduction states.

\section{ELECTRICAL CONDUCTIVITY}

An important application of doped $a-\mathrm{Si}: \mathrm{H}$ is the use of the material as the active focal plane element in infrared imaging (IR) devices. The physical property of the $a-\mathrm{Si}: \mathrm{H}$ that renders it useful for this application is a high temperature coefficient of resistivity (TCR). The physical principal of the device is straightforward: it is conceptually identical to an ordinary optical camera but with optics focusing an IR image onto a high TCR material placed at the focal plane. If this plane is subdivided into pixels, then the local temperature of the individual pixels conveys information about the IR intensity in that part of the image and the pixels taken in their entirety to provide an IR image of the scene. Evidently, there are myriad nontrivial technical aspects to make a real device, but this is the basic idea.

To connect these devices to microscopic theory (wave functions, atomic coordinates, doping levels, etc), the key is to estimate the temperature dependence of the electrical conductivity. Inspection of Fig. 3 shows that the thermal modulation of the Kohn-Sham eigenvalues is largest near the Fermi level and in $a-\mathrm{Si}: \mathrm{H}$ very well correlated with the localization of the states. If we imagine doping such a system - moving the Fermi level into an energy range in which the eigenvalues are strongly influenced by lattice vibrations - one can intuitively expect strong temperature dependence in the conductivity and a high TCR. Quantification of this is made possible by the celebrated work of Kubo on linear response theory [55] merged with first principles methods. The Kubo 
formalism is complicated especially in its full many-body form [56-58], but a simple and graceful result for the AC conductivity emerges with from the single particle approximation of Greenwood [59]. In the Kubo-Greenwood formalism, which is valid in the weak-field limit and for elastic scattering processes, the analytical expression for the AC conductivity is given by [60]:

$$
\begin{aligned}
\sigma_{\alpha \alpha}(\omega) & =\frac{\pi e^{2} \hbar}{\Omega m^{2}} \sum_{n m}\left|\left\langle\psi_{n}\left|\mathbf{p}_{\alpha}\right| \psi_{m}\right\rangle\right|^{2} \frac{f_{F D}\left(\varepsilon_{m}\right)-f_{F D}\left(\varepsilon_{n}\right)}{\hbar \omega} \\
& \times \delta\left(\varepsilon_{n}-\varepsilon_{m}-\hbar \omega\right)
\end{aligned}
$$

where $\alpha=x, y, z, \mathrm{f}_{F D}$ is the Fermi-Dirac distribution, $e$ and $m$ are the electronic charge and mass, $\mathbf{p}_{\alpha}$ is the momentum operator, $\psi_{i}$ and $\varepsilon_{i}$ are the eigenstates and eigenvalues and $\Omega$ is the cell volume. The total AC conductivity is then $\sigma(\omega)=\frac{1}{3} \sum_{\alpha} \sigma_{\alpha \alpha}$. In the dc-limit $(\omega \rightarrow$ $0)$, the real part of the conductivity takes the form,

$$
\sigma(T)=-\frac{1}{3} \sum_{\alpha} \int_{-\infty}^{\infty} \sigma_{\alpha \alpha}(\varepsilon) \frac{\partial f_{F}(\varepsilon)}{\partial \varepsilon} d \varepsilon
$$

where,

$$
\sigma_{\alpha \alpha}(\varepsilon)=\frac{\pi e^{2} \hbar}{\Omega m^{2}} \sum_{n m}\left|\left\langle\psi_{n}\left|p_{\alpha}\right| \psi_{m}\right\rangle\right|^{2} \delta\left(\varepsilon_{n}-\varepsilon\right) \delta\left(\varepsilon_{m}-\varepsilon\right) .
$$

To include thermally-induced electron states and energy fluctuations near the gap, we average the expression in Eq. 17 over a thermal simulation at a fixed temperature. The DC conductivity can be constructed from a trajectory average (this approach is influenced by the work of Allen and Broughton [61]),

$$
\bar{\sigma}_{\alpha \alpha}(\varepsilon)=\frac{\pi e^{2} \hbar}{\Omega m^{2}} \sum_{n m} \overline{\left|\left\langle\psi_{n}^{t}\left|p_{\alpha}\right| \psi_{m}^{t}\right\rangle\right|^{2} \delta\left(\varepsilon_{n}^{t}-\varepsilon\right) \delta\left(\varepsilon_{m}^{t}-\varepsilon\right)}
$$

where the bar denotes the average and we emphasize the dependence of the various terms on the simulation time $t$. This average then picks up thermal broadening effects in the density of states, and also include timedependence in the dipole matrix element. We insert Eq. 18 into Eq. 16 to obtain the temperature-dependent conductivities reported in reference [62].

We see that there is indeed a very strong T-dependence for the conductivity, and the TCR behaves much like experiments on doped a-Si:H, as we report in detail in reference [62].

\section{MEYER-NELDEL RULE}

In a band picture of conduction in semiconductors, one expects an exponential dependence of conductivity with energy, $\sigma=\sigma_{o} \exp \left(-E_{a} / k_{B} T\right)$, where $E_{a}$ is the activation energy and $\sigma_{o}$ is an energy-dependent prefactor.
Meyer proposed a simple relationship between the prefactor $\sigma_{0}$ and the activation energy $E_{a}$,

$$
\ln \sigma_{o}=A+B E_{a}
$$

known as the Meyer-Neldel Rule (MNR) [15]. In view of the equation above, the conductivity $\sigma$ can be written as,

$$
\sigma=\sigma_{o} \exp \left(\frac{-E_{a}}{k_{B} T}\right)=\sigma_{o o} \exp \left(\frac{E_{a}}{E_{\mathrm{MNR}}}\right) \exp \left(\frac{-E_{a}}{k_{B} T}\right)
$$

where $E_{\mathrm{MNR}}$ is a system-specific energy related to $B$ and $\sigma_{o o}$ is a new prefactor. MNR has been found to be followed approximately not only by oxide semiconductors but also for a class of semiconductors including inorganic [63], organic [64, 65], and amorphous semiconductors (for example, $a$-Si:H in connection with light-induced changes of conductivity $[66,67])$.

To understand the origin of the MNR, it is important to understand the role of energy fluctuations in the context of our present problems. For activated processes with activation energy greatly exceeding the characteristic excitation energies available to the system, a fluctuation involving many small excitations is required to push the system over the barrier. The more ways the necessary fluctuation can occur, the more likely it is for the process to succeed. This led to the concept of "Multiple Excitation Entropy" (MEE), which has clarified the origin of the Meyer-Neldel Rule (MNR) in a great many different activated processes [68]. The Kubo-Greenwood formula represents a sum over different (scattering) pathways, in which case the number of available paths or "channels" is temperature dependent and certainly increasing, reflecting an entropic increase as discussed in MEE [69].

By performing a linear fit on the DC conductivity results, we have identified the intercept at $(1 / T)=0$ to $\sigma_{o}$ and the slope to the activation energy $E_{a}$. There are experimental results on $a-\mathrm{Si}: \mathrm{H}$ that give this exponential behavior with $E_{\mathrm{MNR}}=0.067 \mathrm{eV}$ or $T_{\mathrm{MNR}}=864 \mathrm{~K}$ $[70,71]$. By plotting $\sigma$ as a function of $1 / T$ for various dopants ( $n$-type as well as $p$-type), we have extracted $\sigma_{o}$ and $E_{a}$ for $a-\mathrm{Si}_{61} \mathrm{H}_{10}$ and the results are shown in Fig. 7 of Ref. [72]. Our result shows exponential behavior of $\sigma_{o}$ as a function of the $E_{a}$, the Meyer-Neldel rule, with $E_{\mathrm{MNR}}=0.060 \mathrm{eV}$.

The preceding shows that the mechanism underlying MNR is present in our simulations. The increase of conductivity with increasing activation energy can be understood in our picture as being due to the increase in electron-phonon coupling with increasing activation energy (and therefore localization). Since localized states possess an "amplified" electron-phonon coupling as we emphasize in this paper, some compensation is to be expected. In our picture, the MNR arises because the phonons treat electrons with different localization (or $E_{a}$ ) differently, and the effect runs in a direction consistent with experiment: for doping into the more localized states, the electron-phonon coupling is larger and serves to modulate the energies more strongly than for 
more weakly localized states with smaller $E_{a}$. The "compensation" is due to phonons selectively "shaking" more localized electrons. The logic of the process is given by Overhof and Thomas [73], wherein they discuss "phononinduced delocalization".

\section{OPTICAL PROPERTIES BY DESIGN}

In the theory of complex materials, one confronts the following question: "Given particular optical properties (for example, extent of an optical gap or lack thereof), what atomic coordinates provide the desired properties?". This is an inverse problem, and requires a computational scheme that efficiently explores the configuration space and leaves us with an atomistic model satisfying our conditions. A way to approach this problem is to construct a large number of models using some random process, and keep promising candidates. While this can be effective for small systems (crystals with a small unit cell), it is impractical for more complex systems like amorphous materials. In some cases, the emphasis is not to construct a full structural model from scratch, but rather to improve an existing model by imposing additional atomistic information on the model. A simple example is to generate a model of $a-\mathrm{Si}$ with a realistic optical gap from an amorphous silicon network with defect states in the gap region.

There are diverse motivations for these calculations. The most obvious reason is to find materials optimized for photovoltaic (PV) applications, i.e. tuning a band gap to the solar spectrum, for example. It is also possible to induce metallization via gap tuning: compelling the density of states to be large at the Fermi level would significantly increase the conductivity of the model to induce transition to the metallic state. It also provides an effective means to discover the structural signatures of photodarkening or photobleaching, much studied effects in chalcogenide glasses [74]. For phase-change computer memory materials, the optical contrast between the amorphous and crystalline phases is fundamental, and these techniques should enable additional insight into the processes. Finally, we have shown in our initial forays with this scheme that including a priori electronic information has important structural effects: for example, insisting that $a$-Si have a defect-free gap produces models that also agree better with structural measurements.

Following our discussion in the Introduction, the quantity $-\frac{\partial \lambda_{i}}{\partial R_{\alpha}}$ is easy to obtain from any electronic-structurebased simulation scheme using the Hellmann-Feynman theorem. It is also clear that this force points the direction in the configuration space to maximally shift $\lambda_{i}$ to lower electronic energies (or higher if we move in the opposite direction). Thus these gradients provide us a means to purposefully "push" a computer model toward the desired conditions for optimal design of materials' properties. Following Ref. 32, we summarize below the scheme and discuss how to generalize the result for first- principles interactions.

\section{A. Formulation for Orthogonal Tight-binding[32]}

In the tight-binding formulation of electronic structure calculations, the spatially non-local part of the interatomic force, also known as the band-structure force, has the form:

$$
F_{\alpha}^{B S}=-\sum_{i}^{o c c}\left\langle\psi_{i}\left|\frac{\partial H}{\partial R_{\alpha}}\right| \psi_{i}\right\rangle=-\sum_{i}^{o c c} \frac{\partial \lambda_{i}}{\partial R_{\alpha}} \equiv \sum_{i}^{o c c} F_{i, \alpha}^{B S}
$$

If one considers individual terms in the sum in Eq.(21), the term $F_{i}^{B S}$ represents the contribution from the $i^{\text {th }}$ eigenvalue to the total band-structure force. In effect, $F_{i}^{B S}$ is a gradient for the $i^{t h}$ energy eigenvalue $\lambda_{i}$. As such, $F_{i}^{B S}$ provides the direction in the $3 \mathrm{~N}$-dimensional configuration space of most rapid change of $\lambda_{i}$. Thus, to shift $\lambda_{i}$ to higher (lower) energies, we should move atoms incrementally along the direction $-F_{i}^{B S}\left(+F_{i}^{B S}\right)$. For incremental displacements $\delta R_{\alpha}$ along this gradient, the shift $\delta \lambda_{i}$ of an eigenvalue $\lambda_{i}$ can be written as $\delta \lambda_{i}=$ $\sum_{\alpha}-F_{i, \alpha}^{B S} \delta R_{\alpha}$. To this end, we introduce the term gap force for state $i$ to indicate the force (negative nuclear gradient) associated with eigenvalue $\lambda_{i}$. We exploit such forces to push eigenvalues out of a spectral range that we wish to be free of states. Our modified or biased dynamics follows from a Lagrangian $\mathcal{L}=T-\Phi$, in which $T=\frac{1}{2} \sum_{\alpha=1}^{3 N} m_{\alpha} \dot{R}_{\alpha}^{2}$, and

$$
\begin{aligned}
\Phi\left(R_{1}, R_{2} \ldots, R_{3 N}\right) & =\sum_{i} f_{i}\left\langle\psi_{i}|H| \psi_{i}\right\rangle+U_{r} \\
& \left.+\sum_{i}^{\prime} \gamma g\left(\lambda_{i}\right)\left(\left\langle\psi_{i}|H| \psi_{i}\right\rangle-\varepsilon_{f}\right\rangle 22\right)
\end{aligned}
$$

The sum in the last term in Eq. (22) is restricted to an energy range we wish to clear of states. Here, $g\left(\lambda_{i}\right)$ 's are chosen +1 or -1 depending on the desired eigenvalues of these states, and $f_{i}$ is the occupation number of $i^{\text {th }}$ energy level, which is either 0,1 , or 2 . The parameter $\gamma$ controls the strength of the gap force, $\varepsilon_{f}$ is the Fermi energy, and $U_{r}$ is the repulsive ion-ion interaction. The force associated with the $\alpha^{t h}$ degree of freedom is given by,

$$
F_{\alpha}^{b i a s}=F_{\alpha}^{B S}+F_{\alpha}^{i o n}+F_{\alpha}^{g a p},
$$

which can be used to obtain stable local minima by minimizing the total energy and forces via MD simulations and/or relaxations. In the tight-binding formulation, the forces on the right-hand side of Eq. 23 can be explicitly 
written as,

$$
\begin{aligned}
F_{\alpha}^{B S} & =-\sum_{i} f_{i}\left\langle\psi_{i}\left|\frac{\partial H}{\partial R_{\alpha}}\right| \psi_{i}\right\rangle \\
F_{\alpha}^{i o n} & =-\frac{\partial U_{r}}{\partial R_{\alpha}} \\
F_{\alpha}^{g a p} & =-\sum_{i, \lambda_{i}=E_{\min }}^{E_{\max }} \gamma g\left(\lambda_{i}\right)\left\langle\psi_{i}\left|\frac{\partial H}{\partial R_{\alpha}}\right| \psi_{i}\right\rangle
\end{aligned}
$$

We show empirically that the method works well even for midgap states near $\varepsilon_{f}$. We have observed that the method is also applicable in the opposite mode: to maximize the density of states at the Fermi level by shepherding eigenvalues toward the Fermi level. This might, for example, introduce new structural features and produce models with interesting electrical conductivity.

In Refs. [32] and [75], we have demonstrated that the method is quite effective in producing structure with a prescribed optical gap. We used tight-binding parametrizations for $\mathrm{C}$ and $\mathrm{Si}[76,77]$ to conduct MD simulations where we modified the atomic forces towards constructing amorphous structures with prescribed gap. We found that such electronic constraints have profound implications on the structure. For example, we show that we can tune the concentration of tetrahedrally bonded $\left(s p^{3}\right.$ bonded) carbon atoms in a model of amorphous carbon by tuning the magnitude of the gap forces. Note that $s p^{3}$-bonded carbon has wide band gap where as $s p^{2}$-bonded carbon is conducting, and accordingly, we show that a quench-from-the-melt dynamics biased to wider band gap produces models with high concentration of $s p^{3}$-bonded carbon. The magnitude of the gap force can be tuned with parameter $\gamma$ and in doing so we can tune the $s p^{3}$ concentration almost continuously (see figure 17 from Ref. [75]). This constitutes a unique knob in MD simulations where we can smoothly navigate between two distinct phases of $a$-C that have different bond length, coordination and electronic structure.

We have also used gap sculpting to model $a$-Si using tight-binding formulation. Conventional methods of modeling $a$-Si produce models with a few to many geometrical defects, usually in the form of over-coordinated atoms, which in turn register mid-gap states in the DOS of these models. In our calculations, we have inverted the problem and have constrained the MD simulations to have electronic structure with a clean gap. This has produced models that have remarkably fewer defects in the model. For more on the calculations, please refer to $[32,75]$

\section{B. Density functional approach}

Gap sculpting is preferably performed using first principles calculations since tight-binding methods suffer

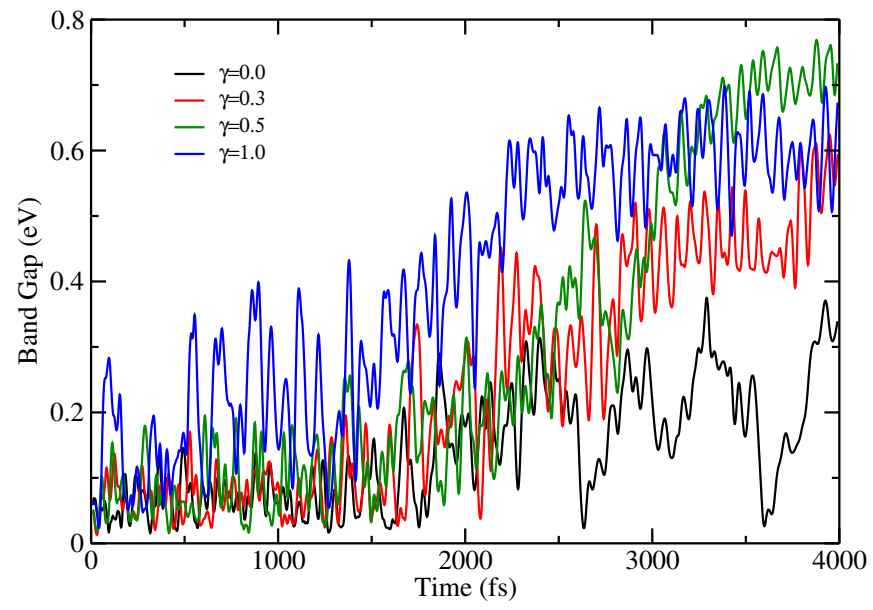

FIG. 16: The band gap of the model during biased quenching from $1800 \mathrm{~K}$ to $300 \mathrm{~K} . \gamma$ represents the biasing factor, which remains constant throughout the quenching dynamics. For all data sets, a running window average is taken to reduce the noise.

from well-known problems of limited transferability. We have used VASP to carry out biased dynamics. ${ }^{11}$ As an early example of the method, we report here a wide band gap amorphous form of silicon that is structurally different from the tetrahedral continuous random network picture of $a$-Si. This structure is stable and has lower energy that its conventional counterpart.

We started with a random collection of 64 atoms and prepared a liquid model of silicon $(l-\mathrm{Si}){ }^{12}$ The liquid model so obtained was then quenched to $300 \mathrm{~K}$ using biased forces. The band gap of the 64-atom WWW model was taken as reference to electronic gap. We tested 4 different biasing factors $(\gamma=0.0,0.3,0.5$ and 1.0). We see that the biased dynamics gradually steers the model to configurations with larger electronic gap and the size of the gap roughly correlates with value of $\gamma$ within reasonable limit (Fig. 16).

The quenched models are then relaxed to their nearest minimum using true LDA forces $(\gamma=0)$. We find that relaxed models so obtained have a band gap larger than conventional model by $0.24 \mathrm{eV}$ (for $\gamma=0.3$ ), $0.35 \mathrm{eV}$ (for $\gamma=0.5$ ) and $0.15 \mathrm{eV}$ (for $\gamma=1.0$ ). The band gap of our best model (that with $\gamma=0.5$ ) is still smaller than that of WWW model of the same volume by $0.25 \mathrm{eV}$. We report the comparison of electronic EDOS and RDF of the model using $\gamma=0.5$ with the conventional model (i.e. using $\gamma=0.0)$. The former has more structural defects

11 The dynamics is biased in the sense that it prefers some minima in energy landscape over others. In particular, the minima that conform to a prescribed band gap are preferred.

12 The system was heated to $1800 \mathrm{~K}$ for $2 \mathrm{ps}$ and was then melted at various temperatures higher than $1800 \mathrm{~K}$ for $10 \mathrm{ps}$. The system was then equilibrated at $1800 \mathrm{~K}$ for another $12 \mathrm{ps}$. 


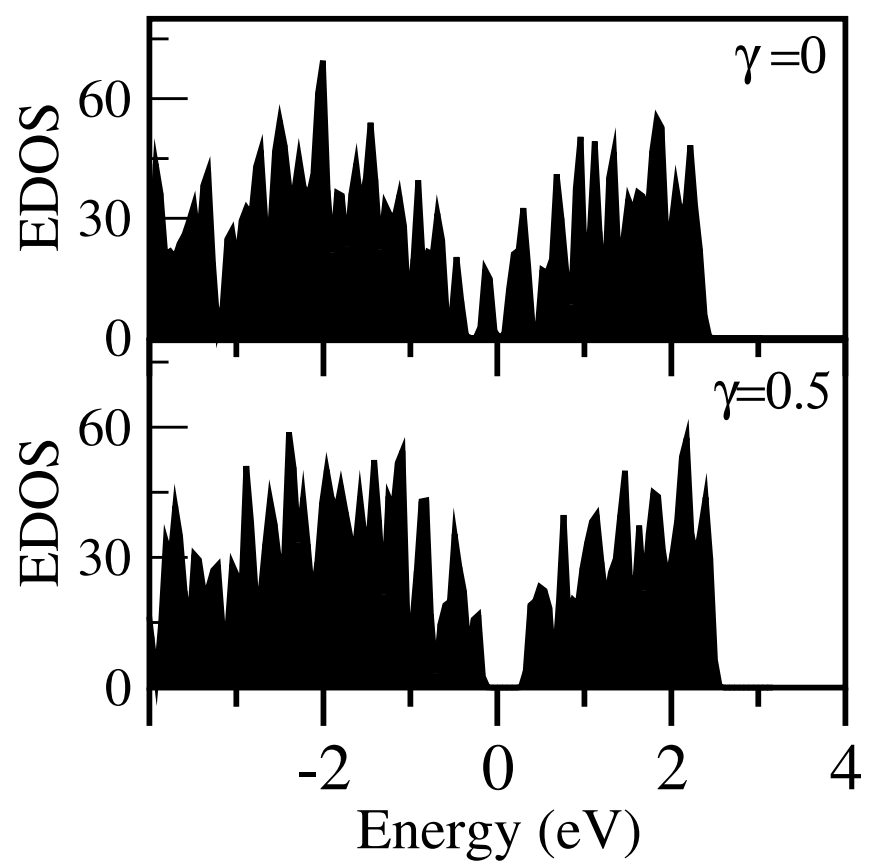

a) Electronic Density of States

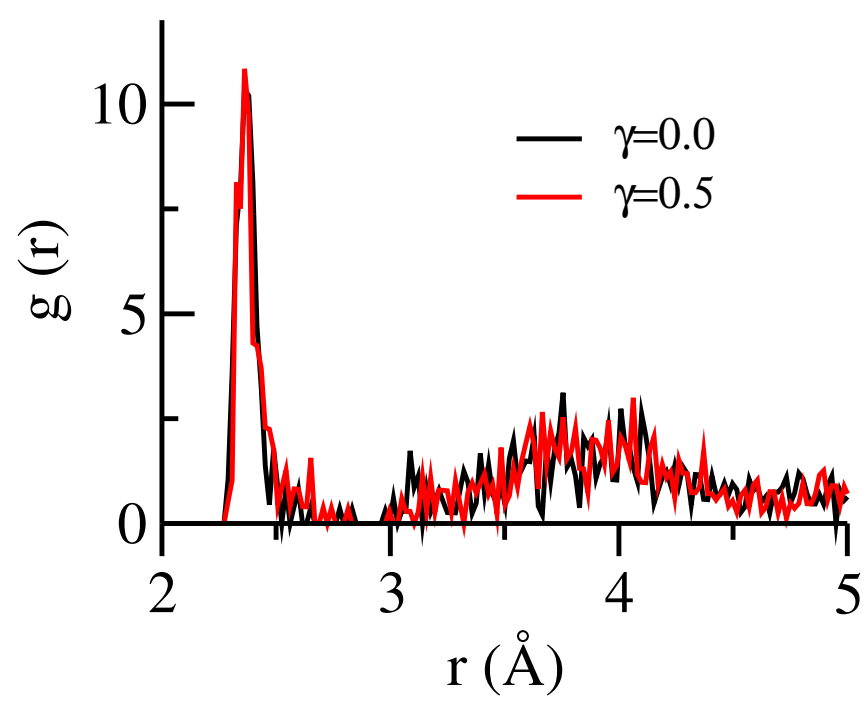

b) Radial Distribution Function

FIG. 17: EDOS and RDF of the relaxed models of $a$-Si. The model indicated $\gamma=0.0$ is constructed using conventional MD where as the one indicated $\gamma=0.5$ is constructed using biased dynamics with $\gamma=0.5$. The $\Gamma$-point gap is $0.64 \mathrm{eV}$ for model with $\gamma=0.5$, compared to $0.89 \mathrm{eV}$ in WWW a-Si of the same volume. The calculation was carried out with the plane-wave LDA code VASP. The Fermi-energy is at $0 \mathrm{eV}$.

in the form of floating bonds than the later and it is remarkable that these defects somehow do not register states in the gap. The structure with $\gamma=0.5$ has lower energy than its conventional counterpart $(\gamma=0)$ by 0.02 $\mathrm{eV} /$ atom but has higher energy than WWW model of same volume by $0.07 \mathrm{eV} /$ atom.

\section{CONCLUSIONS}

In conclusion, we have carried out an array of accurate thermal MD simulations of realistic models of a-Si, a-Se and a-GaN. We have demonstrated a strong correlation between localization and thermally-induced modulation of Kohn-Sham eigenvalues. We report a new correlation (Eq. 14) that works for all three highly distinct materials. The value of this observation lies in its generality across systems with dramatically different chemistry and short-range order. We reveal the effects of changes in cell charge states, as a necessary ingredient to model lightinduced changes in such materials. We next discussed the role of phonons in electronic transport by considering the use of a thermally averaged form of the Kubo-Greenwood formula, and applied the idea to the Meyer-Neldel compensation law. Finally, we took an initial stab at the use of ab initio interactions in our "gap-sculpting" scheme. In this case rather than looking at the effects of phonons on electrons (as in the rest of the paper), we looked at the consequences of applying electronic information in the course of structure modeling. We showed in a small model of a-Si that it is possible to obtain a $\Gamma$ point gap not much different from the best amorphous tetrahedral models of a-Si - but in this case with several defects and reasonable energetics. While this is only a first example, it suggests that the scheme might hold promise for other problems requiring first principles interactions.

The "amplification factor" ( $\alpha$ from Eq. 14) is found to be largest for the conduction states of $a$-Se. For both $a$-Se and $a$-GaN, the linear correlation was very strong and broke into distinct branches for conduction tails. It would be an interesting project to design materials with maximal $\alpha$ - one might expect improved behavior for microbolometer applications, for example.

This paper attempts to answer significant questions but also begs new questions: why is there a strong linear correlation between IPR and thermal variation of eigenvalues? How does $a$-GaN produce an extended state in the gap? This work also reveals the stark differences between crystalline and amorphous materials. As usual, the disordered variants yield new phenomena unknown in crystals and technologies have found ways to exploit these. Doubtless many more remain!

\section{ACKNOWLEDGEMENTS}

We thank the National Science Foundation under the grant numbers DMR 1507670 and 1507166 and the Army Research Office for supporting this work. We also thank the Ohio Supercomputer Center for an allocation of computer time.

We thank Edward Theodoric Drabold for building a computer which carried out some of these calculations and Anup Pandey for helpful advice. 
[1] Misra S, Yu L, Foldyna M and i Cabarrocas P R 2013 Solar Energy Materials and Solar Cells 118 90-95

[2] Fieque B, Tissot J, Trouilleau C, Crastes A and Legras O 2007 Infrared Physics \& Technology 49 187-191

[3] Street R A 2005 Hydrogenated amorphous silicon (Cambridge University Press)

[4] Koelmans W W, Sebastian A, Jonnalagadda V P, Krebs D, Dellmann L and Eleftheriou E 2015 Nature communications 6

[5] Optical fiber URL https://en.wikipedia.org/wiki/ Optical_fiber

[6] Drabold D, Abtew T, Inam F and Pan Y 2008 Journal of Non-Crystalline Solids $\mathbf{3 5 4} 2149-2154$

[7] Fedders P and Drabold D 1996 Physical Review B 53 3841

[8] Zhang X and Drabold D 2001 Physical Review B 63 233109

[9] Stephan U and Drabold D A 1998 Physical Review B 57 6391

[10] Taraskin S, Drabold D and Elliott S 2002 Physical review letters $\mathbf{8 8} 196405$

[11] Weaire D and Thorpe M F 1971 Physical Review B 4 2508

[12] Anderson P W 1958 Physical review 1091492

[13] Dong J and Drabold D 1998 Physical review letters 80 1928

[14] Ludlam J, Taraskin S, Elliott S and Drabold D 2005 Journal of Physics: Condensed Matter 17 L321

[15] Meyer W and Neldel H 1937 Z. tech. Phys 18 588-593

[16] Staebler D and Wronski C 1977 Applied Physics Letters 31 292-294

[17] Carlsson A 1990 Solid State Physics: Adv. in Research and Applications 431

[18] Sankey O F and Allen R E 1986 Physical Review B 33 7164

[19] Sankey O F and Niklewski D J 1989 Physical Review B 403979

[20] Car R and Parrinello M 1985 Physical review letters 55 2471

[21] Drabold D A, Fedders P, Klemm S and Sankey O F 1991 Physical review letters $\mathbf{6 7} 2179$

[22] Jain A, Ong S P, Hautier G, Chen W, Richards W D, Dacek S, Cholia S, Gunter D, Skinner D, Ceder G et al. 2013 Apl Materials 1011002

[23] Jones R O 2015 Reviews of Modern Physics 87897

[24] Martin R M 2004 Electronic structure: basic theory and practical methods (Cambridge university press)

[25] Drabold D and Fedders P 1999 Physical Review B 60 R721

[26] Runge E and Gross E K 1984 Physical Review Letters 52 997

[27] Li J and Drabold D 2003 Physical Review B 68033103

[28] Hybertsen M S and Louie S G 1986 Physical Review B 345390

[29] Kresse G and Furthmüller J 1996 PRB 5411169

[30] Kresse G and Furthmüller J 1996 Computational Materials Science 6 15-50

[31] Zhang X and Drabold D 1999 Physical Review Letters 83 5042

[32] Prasai K, Biswas P and Drabold D 2015 Scientific reports 515522
[33] Drabold D, Dow J, Fedders P, Carlsson A and Sankey O F 1990 Physical Review B 425345

[34] Drabold D and Fedders P 1999 Physical Review B 60 R721

[35] Urbach F 1953 Physical Review 921324

[36] Pan Y, Inam F, Zhang M and Drabold D 2008 Physical review letters 100206403

[37] Aljishi S, Cohen J D, Jin S and Ley L 1990 Physical review letters $\mathbf{6 4} 2811$

[38] Dong J and Drabold D 1996 Physical Review B 5410284

[39] Drabold D 2000 J. Non-Crystalline Solids 266211

[40] Atta-Fynn R, Biswas P and Drabold D 2004 PRB 69 245204

[41] Mahan G Many particle physics,(plenum press, new york, 1990)

[42] Feynman R P 1939 Physical Review 56340

[43] Sharma R, Prasai K, Drabold D and Adarsh K 2015 AIP Advances $\mathbf{5} 077164$

[44] Fedders P, Fu Y and Drabold D 1992 Physical review letters $\mathbf{6 8} 1888$

[45] Abtew T and Drabold D 2005 Journal of Physics: Condensed Matter 18 L1

[46] Drabold D, Zhang X and Li J 2002 Photo-Induced Metastability in Amorphous Semiconductors 260-278

[47] Li J and Drabold D 2000 Physical review letters 852785

[48] Kastner M, Adler D and Fritzsche H 1976 Physical Review Letters 371504

[49] Street R and Mott N 1975 Physical Review Letters 35 1293

[50] Wooten F, Winer K and Weaire D 1985 Physical review letters $\mathbf{5 4} 1392$

[51] Djordjević B R, Thorpe M F and Wooten F 1995 PRB $\mathbf{5 2} 5685$

[52] Inam F, Lewis J P and Drabold D 2010 physica status solidi (a) 207 599-604

[53] Cai B and Drabold D 2011 Physical Review B 84075216

[54] Stumm P and Drabold D 1997 Physical review letters 79 677

[55] Kubo R 1957 Journal of the Physical Society of Japan $12570-586$

[56] Zhang M and Drabold D 2011 Journal of Physics: Condensed Matter 23085801

[57] Zhang M L and Drabold D 2010 Physical review letters 105186602

[58] Zhang M and Drabold D 2010 Physical Review E 83 012103

[59] Greenwood D 1958 Proceedings of the Physical Society 71585

[60] Mott N F and Davis E A 1979 Electronic processes in non-crystalline materials (Oxford University Press, Oxford)

[61] Allen P B and Broughton J Q 1987 Journal of Physical Chemistry 91 4964-4970

[62] Abtew T, Zhang M and Drabold D 2007 Physical Review B 76045212

[63] Busch G 1950 Zeitschrift für Angewandte Mathematik und Physik (ZAMP) $13-31$

[64] Gutmann F and Lyons L E 1967 Organic semiconductors (Wiley)

[65] Rosenberg B, Bhowmik B B, Harder H C and Postow E 1968 Journal of Chemical Physics 49 4108-4114 
[66] Anderson D and Spear W 1977 Philosophical Magazine 36 695-712

[67] Staebler D and Wronski C R 1980 Journal of Applied Physics 51 3262-3268

[68] Yelon A, Movaghar B and Crandall R 2006 Reports on Progress in Physics 691145

[69] Yelon A and Movaghar B 1990 Physical review letters 65 618

[70] Carlson D 1986 Applied Physics A 41 305-309

[71] Fritzsche H 2001 Annual Review of Materials Research 31 47-79

[72] Abtew T, Zhang M, Pan Y and Drabold D 2008 Journal of Non-Crystalline Solids 354 2909-2913

[73] Overhof H and Thomas P 1989 Springer Tracts in Modern Physics $\mathbf{1 1 4}$

[74] Tanaka K and Odajima A 1982 Solid State Communications 43 961-964

[75] Prasai K, Biswas P and Drabold D A 2016 Phys. Status Solidi A 1-8 /DOI 10.1002/pssa.201532973

[76] Xu C, Wang C, Chan C and Ho K 1992 J. Phys Cond. Matt. 46047

[77] Goodwin L, Skinner A and Pettifor D 1989 EPL 9701 